\title{
Molecular determinants for lymph node metastasis in clinically early-stage endometrial cancer
}

\author{
NADIM BOU ZGHEIB ${ }^{1}$, DOUGLAS C. MARCHION ${ }^{1,2}$, STEPHEN H. BUSH $^{1}$, \\ PATRICIA L. JUDSON ${ }^{1-3}$, ROBERT M. WENHAM ${ }^{1-3}$, SACHIN M. APTE ${ }^{1,3}$, \\ JOHNATHAN M. LANCASTER ${ }^{1-3}$ and JESUS GONZALEZ-BOSQUET ${ }^{4}$ \\ ${ }^{1}$ Department of Women's Oncology, ${ }^{2}$ Chemical Biology and Molecular Medicine Program, and \\ ${ }^{3}$ Department of Oncologic Sciences, H. Lee Moffitt Cancer Center and Research Institute, Tampa, FL 33612; \\ ${ }^{4}$ Gynecologic Oncology, Department of Obstetrics and Gynecology, University of Iowa Hospital and Clinics, \\ Iowa, IA 52242, USA
}

Received October 17, 2014; Accepted July 16, 2015

DOI: $10.3892 / \mathrm{ol} .2015 .3883$

\begin{abstract}
Patients with occult lymph node metastasis in endometrioid-type endometrial cancer (EC) are prone to the development of recurrences and have worse outcomes compared with patients without lymph node metastasis. In the current study, the aim was to identify molecular parameters associated with lymph node metastasis in EC clinically early-stage disease. A univariate analysis of differentially expressed genes, proteins and clinicopathological parameters (including myometrial invasion and tumor grade) was performed, comparing EC patients with and without lymph node metastasis $(n=262$ patients from The Cancer Genome Atlas). Significant parameters were introduced in a multivariate model and a gene expression pathway analysis. Lymph node metastasis was associated with expression of 268 unique genes $(\mathrm{P}<0.001), 19$ unique proteins $(\mathrm{P}<0.05)$, tumor grade and myometrial invasion in univariate analysis. Multivariate analysis demonstrated 10 genes independently associated with lymph node metastasis and 4 independently associated proteins. Myometrial invasion was the only independent clinicopathological parameter associated with lymph node status. The enrichment pathway analysis demonstrated that expression of epidermal growth factor receptor, $\mathrm{Bcl} 2$ antagonist of cell death and phosphatase and tensin homolog pathways were significantly involved in lymph node metastasis ( $\mathrm{P} \leq 0.001)$. A gene expression signature to predict lymph node status in EC was created for future validation. Few studies have focused on the association between EC's molecular characteristics
\end{abstract}

Correspondence to: Dr. Sachin M. Apte, Department of Women's Oncology, H. Lee Moffitt Cancer Center and Research Institute, 12902 Magnolia Drive, Tampa, FL 33612, USA

E-mail: sachin.apte@moffitt.org

Key words: endometrial cancer, clinicopathological and molecular parameters, lymph node metastasis and nodal metastasis. Defining molecular risk factors for EC lymphatic nodal metastasis may help to individualize treatment and improve patient outcomes.

\section{Introduction}

Endometrial cancer (EC) is the most common gynecological malignancy, with nearly 50,000 new cases diagnosed each year in the USA (1). Although the majority of EC patients present with early-stage, curable disease, a significant subset present with advanced-stage or develop recurrent disease that is associated with a less favorable outcome (1). Currently, the prognosis and survival of patients with EC have been largely indicated by the information obtained during surgery (2). A significant number of clinically early-stage cases have extrauterine disease within the pelvic and paraaortic lymph nodes, which are the most common sites for metastasis. The risk of pelvic lymph node metastasis for clinical stage I EC has been associated with tumor grade and the depth of myometrial invasion (2). A Gynecologic Oncology Group Study reported that patients with poorly differentiated cancers or who have cancer invading the outer half of the myometrium have a $>10 \%$ risk of pelvic lymph node metastasis (2). However, in that study, 22\% of clinical stage I cancers had extrauterine disease. Although many clinicians believe that nodal dissections must be reserved for those with sufficient risk of nodal metastasis (2-5), the risk level of nodal disease that warrants this procedure is unclear. The major risks associated with nodal dissection include increased operative time, potential for blood loss associated with vascular injury, genitofemoral nerve injury, lymphocyst formation and lymphedema (3,6-8).

Selective surgical staging allows the identification of patients with lymph node metastasis and avoids the morbidity of routine lymphadenectomy. Although a number of preoperative and intraoperative risk factors for nodal metastasis have been identified, they have a low positive predictive value in clinical practice. Preoperative radiological testing appears to identify patients with a low rather than high risk of nodal metastasis. Furthermore, ultrasound (9), computed tomography (10) and magnetic resonance imaging (11) have 
Table I. Characteristics of patients with available genetic expression data $(\mathrm{n}=203)$.

\begin{tabular}{|c|c|c|c|}
\hline Characteristics & Lymph node positive $(\mathrm{n}=38)$ & Lymph node negative $(\mathrm{n}=165)$ & P-value \\
\hline Age, years (mean) & 62.6 & 62.7 & 0.98 \\
\hline Menopausal status, $\%$ & & & 0.73 \\
\hline Premenopausal & 8.8 & 7.3 & \\
\hline Postmenopausal & 91.2 & 92.7 & \\
\hline Surgical approach, \% & & & 0.08 \\
\hline Laparotomy & 81.6 & 67.3 & \\
\hline Minimally invasive & 18.4 & 32.7 & \\
\hline Body mass index (mean) & 33.6 & 32.1 & 0.29 \\
\hline Depth of invasion, \% (mean) & 65.0 & 37.6 & $<0.01$ \\
\hline Grade, $\%$ & & & $<0.01$ \\
\hline 1 & 5.3 & 27.9 & \\
\hline 2 & 7.9 & 33.9 & \\
\hline 3 & 86.8 & 38.2 & \\
\hline Peritoneal washings, $\%$ & & & 1.00 \\
\hline Positive & 12.5 & 12.8 & \\
\hline Negative & 87.5 & 87.2 & \\
\hline
\end{tabular}

been found to be suboptimal in identifying patients with pelvic or paraaortic nodal metastases. The identification of an accurate test to predict lymph node metastasis in patients with EC would have substantial clinical application. The present study sought to identify the molecular and clinicopathological markers determining lymph node metastasis. These markers will be the basis for a predictive model for lymph node metastasis in EC.

\section{Materials and methods}

Databases used for the study. The Cancer Genome Atlas (TCGA) database of the National Cancer Institute (Bethesda, MD, USA) was used for the current study (http://cancergenome. nih.gov/). Data were collected in August 2012. All cases of EC with information regarding lymph node status were selected for inclusion. TCGA gene expression, protein expression and clinicopathological data for EC patients were downloaded. The clinicopathological parameters included tumor grade, depth of myometrial invasion and lymph node status. TCGA has collected $>373$ EC samples with gene expression (mRNA) data as well as clinical information (12).

Statistical analysis. Analysis of gene expression was performed using Biometric Research Branch (BRB) ArrayTools (Version 2.13.2 for x64 systems), an integrated package for visualization and statistical analysis that utilizes Excel (Microsoft, Redmond, WA) as front-end, and tools developed in the R statistical system. BRB-ArrayTools were developed by Dr Richard Simon and the BRB-ArrayTools development team (http://linus.nci.nih.gov/BRB-ArrayTools/). Additional analyses were performed using the $\mathrm{R}$ statistical software environment for statistical computing and graphics (http://www.r-project.org/), including Bioconductor, an open source software for bioinformatics (http://bioconductor.org/).
Two strategies were used to design a predictor for lymph node metastasis in EC patients: A gene expression signature to predict lymph node status and significantly independent molecular, clinical and pathological variables predicting lymph node status.

Gene expression signature. To construct a gene signature profile that would classify patients into lymph node positive and lymph node negative groups, the Class Prediction Tool of BRB-ArrayTools was used. This tool optimizes the significance level threshold used for gene selection. Genes that were differentially expressed between the classes at a univariate significance level of $<0.001$ were included in the predictor. A number of methods are included in the tool to evaluate the predictor or gene signature. To assess how accurately the groups are predicted by this multivariate class predictor (internal validation), a cross-validated misclassification rate is computed, usually in the form of the leave-one-out cross-validation method (13).

Independent variables predicting lymph node status. A univariate two-tailed $t$-test analysis was performed using lymph node status and gene expression, protein expression and clinicopathological parameters. The nominal significance level of each univariate test was $\mathrm{P}<0.001$. For the gene expression comparison, 10,000 random permutations were performed to determine the probability of ascertaining significant differential expression by chance parameters at the $\mathrm{P}<0.001$ level. Significantly associated parameters were subjected to a multivariable analysis to determine independently associated molecular markers.

Subsequently, independent molecular markers were uploaded to GeneGo MetaCore ${ }^{\mathrm{TM}}$ for pathway analysis (http://www.genego.com/metacore.php; Thomson Reuters, New York, NY) to identify biological processes that may participate in lymph node invasion in EC. Pathways with $\mathrm{P}<0.05$ 
Table II. Characteristics of patients with available protein expression $(n=165)$.

\begin{tabular}{|c|c|c|c|}
\hline Characteristics & Lymph node positive $(\mathrm{n}=26)$ & Lymph node negative $(\mathrm{n}=139)$ & P-value \\
\hline Age, years (mean) & 62.3 & 62.8 & 0.89 \\
\hline Menopausal status, $\%$ & & & 0.67 \\
\hline Premenopausal & 8.0 & 6.5 & \\
\hline Postmenopausal & 92.0 & 93.5 & \\
\hline Surgical approach, $\%$ & & & 0.02 \\
\hline Laparotomy & 92.4 & 67.4 & \\
\hline Minimally invasive & 7.6 & 32.6 & \\
\hline Body mass index (mean) & 31.9 & 33.1 & 0.45 \\
\hline Depth of invasion, \% (mean) & 68.6 & 37.2 & $<0.01$ \\
\hline Grade, $\%$ & & & $<0.01$ \\
\hline 1 & 7.7 & 32.4 & \\
\hline 2 & 11.5 & 36.7 & \\
\hline 3 & 80.8 & 30.9 & \\
\hline Peritoneal washings, $\%$ & & & 0.73 \\
\hline Positive & 15.0 & 13.3 & \\
\hline Negative & 85.0 & 86.7 & \\
\hline Stage, $\mathrm{n}$ & & & $<0.01$ \\
\hline 1 & 0 & 113 & \\
\hline 2 & 0 & 11 & \\
\hline 3 & 22 & 11 & \\
\hline 4 & 4 & 4 & \\
\hline Overall survival time (days) & 764 & 928 & 0.34 \\
\hline Histology, n & & & $<0.01$ \\
\hline Serous & 9 & 13 & \\
\hline Non-serous & 17 & 126 & \\
\hline
\end{tabular}

were considered significant, based on the GeneGo MetaCore ${ }^{\mathrm{TM}}$ statistical test for significance.

\section{Results}

Database analysis results. Using publicly available data for EC collected in TCGA database, 262 patients with lymph node status and clinical information were identified. Of these, 203 patients had available information on genomic expression data and lymph node status and 165 patients had available information on protein data and lymph node status. The 203 patients with available genomic expression data were divided according to their lymph node status, with 165 patients having negative lymph nodes and 38 having positive lymph nodes. These groups were similar with regard to age, menopausal status, peritoneal washing status and body mass index (Table I). Furthermore, of the 165 patients with available protein expression data, 139 were identified to have negative lymph nodes whilst 26 had positive lymph nodes. These groups were also similar with regard to age, menopausal status, peritoneal washing status, overall survival and body mass index (Table II).

Gene expression signature. The class prediction tool identified a gene expression signature composed of 295 genes (Fig. 1A).
Table III. Univariate analysis of protein and clinicopathological data.

\begin{tabular}{lr}
\hline Characteristics & P-value \\
\hline Invasion & $<0.01$ \\
Grade & $<0.01$ \\
BCL-2-M-V & 0.03 \\
Dvl3-R-V & $<0.01$ \\
eEF2K-R-V & 0.04 \\
EGFR-R-C & 0.03 \\
EGFR_pY1068-R-V & 0.01 \\
EGFR_pY1173-R-C & $<0.01$ \\
FOXO3a_pS318_S321-R-C & $<0.01$ \\
HER3_pY1298-R-C & 0.02 \\
Notch1-R-V & 0.01 \\
p21-R-C & 0.02 \\
p70S6K_pT389-R-V & 0.02 \\
PDK1_pS241-R-V & 0.01 \\
PR-R-V & 0.04 \\
Shc_pY317-R-NA & $<0.01$ \\
Smad4-M-V & 0.02 \\
Src_pY527-R-V & 0.03 \\
YB-1-R-V & 0.02 \\
\hline
\end{tabular}


Table IV. Statistically significant results from multivariate analysis of genes, proteins, and clinical variables.

\begin{tabular}{llr}
\hline Characteristics & Standard error & t-value \\
\hline Clinicopathological variables & & 5.115 \\
Invasion & $6.12 \times 10^{-4}$ & \\
Differentially expressed genes & & -2.802 \\
ARSI & $2.39 \times 10^{-4}$ & -4.644 \\
RNF183 & $2.41 \times 10^{-4}$ & 3.321 \\
DNER & $2.37 \times 10^{-4}$ & -2.934 \\
DUSP9 & $2.47 \times 10^{-4}$ & -3.171 \\
TEX19 & $2.62 \times 10^{-4}$ & -3.036 \\
RPS6KA6 & $2.84 \times 10^{-4}$ & -2.970 \\
FBN3 & $2.52 \times 10^{-4}$ & -2.846 \\
MUC6 & $2.15 \times 10^{-4}$ & 3.477 \\
GABRQ & $3.30 \times 10^{-4}$ & $<0.01$ \\
FLJ16779 & $2.54 \times 10^{-4}$ & $<0.01$ \\
Independently associated proteins & & $<0.01$ \\
EF2K & 3.791 \\
EGFR & $3.42 \times 10^{-4}$ & $<0.01$ \\
PDK1 & $3.38 \times 10^{-4}$ & $<0.01$ \\
YB & $2.93 \times 10^{-4}$ & 0.01 \\
\hline PRSI & $3.51 \times 10^{-4}$ & 3.705 \\
\end{tabular}

$A R S I$, arylsulfatase I; RNF183, ring finger protein 183; DNER, delta/notch-like EGF repeat containing; DUSP9, dual specificity phosphatase 9; TEX19, testis expressed 19; RPS6KA6, ribosomal protein S6 kinase, 90kDa, polypeptide 6; FBN3, fibrillin 3; MUC6, mucin 6; $G A B R Q$, gamma-aminobutyric acid A receptor, theta; EF2K, elongation factor 2 kinase; EGFR, epidermal growth factor receptor; PDK1, phosphoinositide-dependent kinase 1; YB, Y box binding protein.
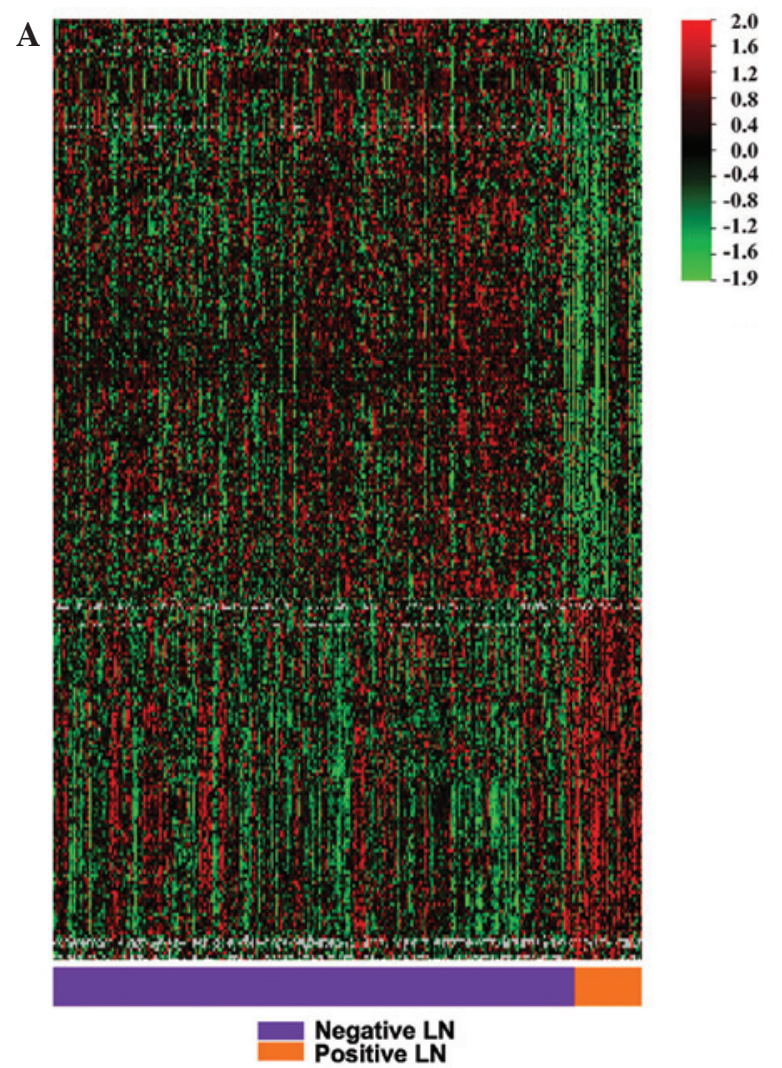

B

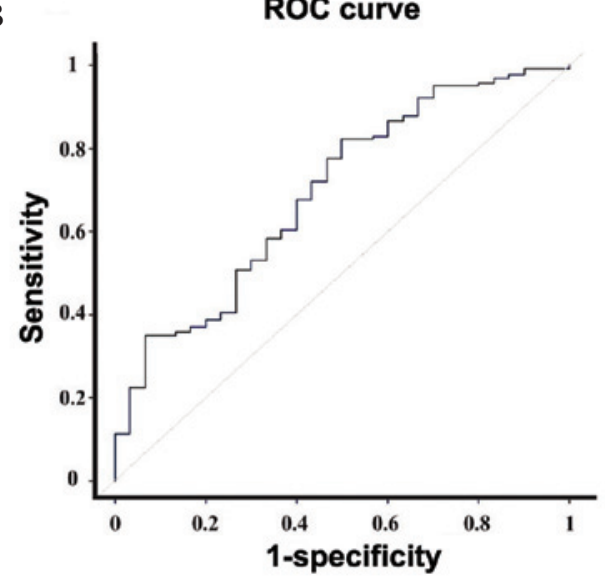

C

\begin{tabular}{|l|c|c|c|}
\cline { 2 - 4 } \multicolumn{1}{c|}{} & \multicolumn{2}{c|}{ TCGA } & \\
\hline Model & Neg & Pos & Total \\
\hline Neg & $\mathbf{1 8 6}$ & $\mathbf{1 5}$ & $\mathbf{2 0 1}$ \\
\hline Pos & $\mathbf{4 6}$ & $\mathbf{1 5}$ & $\mathbf{6 1}$ \\
\hline Total & $\mathbf{2 3 2}$ & $\mathbf{3 0}$ & $\mathbf{2 6 2}$ \\
\hline
\end{tabular}

Figure 1. Gene expression signature as predictor of lymph node (LN) status. (A) Heatmap of the signature predictive of lymph node status. (B) Performance of the model in the training dataset measured by the receiver operating characteristic (ROC) curve. (C) Contingency table of level of agreement between TCGA data and the predictive model: kappa coefficient of 0.2. Patients classified correctly (Neg-Neg and Pos-Pos) are on blue background. Neg, patients with negative lymph node status; Pos, patients with positive lymph node status. 


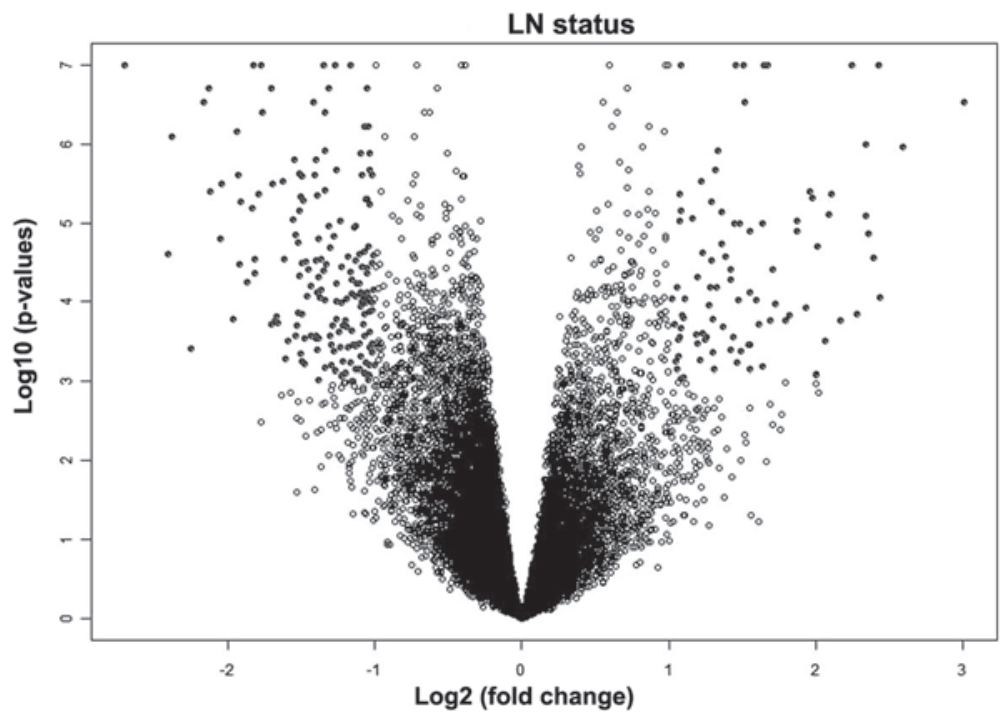

Figure 2. Volcano plot of significant genes from univariate analysis. LN, lymph node. Black points represent significant genes; white points represent nonsignificant genes.

This signature or model correctly predicted lymph node status in the current patient group at a rate of $77 \%$, with $\mathrm{P}=0.04$ (internal validation with the compound covariate predictor). The model was more accurate in predicting negative lymph node status, with a sensitivity of $80 \%$ and a specificity of $50 \%$. The $\kappa$ coefficient was 0.20 , indicating fair agreement between the model and the surgical result (Fig. 1C). The performance of the model, measured by the area under the receiver operating characteristic (ROC) curve, was $70 \%$ (Fig. 1B).

Independent variables predicting lymph node status. The univariate analysis test using lymph node status and EC gene expression and protein expression data identified 268 unique genes $(\mathrm{P} \leq 0.001$; Fig. 2$)$ and 19 unique proteins $(\mathrm{P}<0.05$; Table III) as associated with lymph node metastasis. When the clinicopathological parameters were evaluated with respect to lymph node status, only tumor grade $(\mathrm{P}=0.0003)$ and depth of myometrial invasion $\left(\mathrm{P}=8.15 \times 10^{-6}\right.$; Table III) were determined to be significantly associated with lymph node metastasis.

Based on these findings, the unique genes, proteins and clinicopathological parameters determined to be significantly associated with lymph node metastasis were subjected to a multivariable analysis. This identified 10 genes [arylsulfatase I (ARSI); ring finger protein 183 (RNF183); delta/notch-like EGF repeat containing (DNER); dual specificity phosphatase 9 (DUSP9); testis expressed 19 (TEX19); ribosomal protein S6 kinase, 90kDa, polypeptide 6 (RPS6KA6); fibrillin 3 (FBN3); mucin 6 (MUC6); gamma-aminobutyric acid A receptor, theta $(G A B R Q)$; and $F L J 16779)$ and 4 proteins [elongation factor 2 kinase (EF2K); epidermal growth factor receptor (EGFR); phosphoinositide-dependent kinase 1 (PDK1); and $\mathrm{Y}$ box binding protein (YB)] that were independently associated with lymph node metastasis (Table IV). Interestingly, depth of myometrial invasion was the only clinicopathological parameter to be independently associated with lymph node metastasis. The genes determined to be independently associated with lymph node metastasis $(n=10)$ were uploaded into GeneGo MetaCore ${ }^{\mathrm{TM}}$ pathway analysis, which identified
3 molecular signaling pathways associated with lymph node metastasis $(\mathrm{P}<0.0001)$. These molecular signaling pathways included the expression of $E G F R\left(\mathrm{P}=4.23 \times 10^{-5}, 2 / 23\right.$ pathway objects), $\mathrm{Bcl} 2$ antagonist of cell death $\left(B A D ; \mathrm{P}=1.44 \times 10^{-4}, 2 / 42\right.$ pathway objects), and phosphatase and tensin homolog (PTEN; $\mathrm{P}=1.73 \times 10^{-4}, 2 / 46$ pathway objects) pathways.

\section{Discussion}

The precise molecular events that occur during the development, invasion and formation of metastasis in EC are largely uncharacterized and remain poorly understood (14). In the present analysis, an in silico genome-wide approach was employed to define the molecular underpinnings of lymph node metastasis in EC. Using TCGA database, a number of genes, proteins, and molecular signaling pathways associated with EC lymph node metastasis were identified, and these classifiers were included with the clinicopathological parameters to be able to generate a predictive test for lymph node metastasis.

The objective of TCGA is the collection and processing of biospecimens that may be used for cancer diagnosis and analysis. The biospecimens collected from these cancers meet a stringent set of quality criteria, enabling extracted DNA and RNA to be used for advanced genomic analysis and sequencing technologies. The present analysis included a highly stringent level of statistical testing for associations between clinicopathological parameters, gene and protein expression levels and lymph node metastasis in EC. This stringent statistical methodological approach accounts for a potential bias in genomic datasets and ensures that generated P-values may be interpreted as significant at a relative, as well as an absolute, level.

Ten genes were determined to be independently associated with lymph node metastasis in EC: ARSI, RNF183, DNER, DUSP9, TEX19, RPS6KA6, FBN3, MUC6, GABRQ and FLJ16779. Notably, the ring finger RNF183 gene has been previously reported to be differentially expressed in EC (15). 
In addition, the DNER gene was found to regulate glioblastoma-derived neurosphere cell differentiation and tumor progression (16). RPS6KA6, a putative tumor suppressor gene expressed in normal endometrial tissue, was demonstrated to be silenced via hypermethylation in EC cell lines; however, its role as a suppressor in EC remains uncertain (17). MUC6 gene expression was documented in pancreatic carcinomas and cholangiocarcinomas and focally in endocervical adenocarcinomas (18).

The multivariate analysis identified 4 proteins independently associated with lymph node metastasis in EC: EF2K, EGFR, PDK1, and YB. EGFR is the prototypic member of the ErbB/HER receptor tyrosine kinase family and binds to multiple ligands, including epidermal growth factor, transforming growth factor $\alpha$ and amphiregulin. EGFR is crucial in cellular functions implicated in cancer development (19) and has been revealed to be expressed in a large percentage of endometrial tumors (20).

PDK1 is a key regulator of the AGC protein kinase family, which includes the proto-oncogene $\mathrm{AKT}$ /protein kinase $\mathrm{B}$ implicated in a number of malignancies, including breast cancer. YB-1 appears to play a critical role in cell proliferation and growth, DNA replication, cell cycle and drug resistance, as well as malignancy. Furthermore, YB-1 is overexpressed in cisplatin-resistant cancer cell lines $(13,21)$.

The current data demonstrated an association between lymph node metastasis and a number of gene expression pathways in EC: EGFR, BAD, and PTEN. EGFR has been shown to be a principal growth-promoting pathway in EC cells (22). The $B A D$ pathway influences EC cell sensitivity to cisplatin, likely via modulation of the phosphorylation status of the BAD protein (23). PTEN is the most commonly mutated gene identified in endometrial carcinoma. This mutation is considered to be an early event in endometrial carcinogenesis (24).

The clinical heterogeneity of EC is likely a reflection of an underlying molecular heterogeneity. As such, the mechanisms by which EC cells metastasize are likely to be equally diverse. In the present study, a genome-wide strategy was adopted to characterize some of the molecular signaling pathways and cellular processes that are associated with lymph node metastasis in EC. Such findings may have substantial implications for future clinical treatment of patients with this disease. Empirical treatment based on one-size-fits-all could be replaced with a more tailored therapy that matches the right patient with the right treatment plan based on their own molecular fingerprint. The next phase of this study will be to evaluate and validate the ability of a signature containing independent molecular and clinicopathological parameters to predict lymph node metastasis in patients with EC.

\section{Acknowledgements}

The authors would like to thank the Jacquie Liggett Hearing the Ovarian Cancer Whisper for their fellowship grant support and Rasa Hamilton (Moffitt Cancer Center) for editorial assistance.

\section{References}

1. Siegel R, Ma j, Zou Z and Jemal A: Cancer statistics, 2014. CA Cancer J Clin 64: 9-29, 2014
2. Creasman WT, Morrow CP, Bundy BN, Homesley HD, Graham JE and Heller PB: Surgical pathologic spread patterns of endometrial cancer. A Gynecologic Oncology Group Study. Cancer 60: 2035-2041, 1987.

3. Morrow CP, Bundy BN, Kurman RJ, Creasman WT, Heller P, Homesley HD and Graham JE: Relationship between surgical-pathological risk factors and outcome in clinical stage I and II carcinoma of the endometrium: A gynecologic oncology group study. Gynecol Oncol 40: 55-65, 1991.

4. Faught W, Krepart GV, Lotocki R and Heywood M: Should selective paraaortic lymphadenectomy be part of surgical staging for endometrial cancer? Gynecol Oncol 55: 51-55, 1994.

5. Kim YB and Niloff JM: Endometrial carcinoma: Analysis of recurrence in patients treated with a strategy minimizing lymph node sampling and radiation therapy. Obstet Gynecol 82: 175-180, 1993.

6. Orr JW Jr, Holimon JL and Orr PF: Stage I corpus cancer: Is teletherapy necessary? Am J Obstet Gynecol 176: 777-788; discussion 788-779, 1997.

7. Homesley HD, Kadar N, Barrett RJ and Lentz SS: Selective pelvic and periaortic lymphadenectomy does not increase morbidity in surgical staging of endometrial carcinoma. Am J Obstet Gynecol 167: 1225-1230, 1992.

8. Abu-Rustum NR, Alektiar K, Iasonos A, Lev G, Sonoda Y, Aghajanian C, Chi DS and Barakat RR: The incidence of symptomatic lower-extremity lymphedema following treatment of uterine corpus malignancies: A 12-year experience at memorial Sloan-Kettering cancer center. Gynecol Oncol 103: 714-718, 2006.

9. Cheng WF, Chen CA, Lee CN, Chen TM, Huang KT, Hsieh CY and Hsieh FJ: Preoperative ultrasound study in predicting lymph node metastasis for endometrial cancer patients. Gynecol Oncol 71: 424-427, 1998.

10. La Fianza A, Di Maggio EM, Preda L, Coscia D, Tateo S and Campani R: Clinical usefulness of CT in the treatment of stage I endometrial carcinoma. Radiol Med 93: 567-571, 1997.

11. Frei KA, Kinkel K, Bonél HM, Lu Y, Zaloudek C and Hricak H: Prediction of deep myometrial invasion in patients with endometrial cancer: Clinical utility of contrast-enhanced MR imaging-a meta-analysis and Bayesian analysis. Radiology 216: 444-449, 2000

12. Cancer Genome Atlas Research Network: Kandoth C, Schultz N, Cherniack AD, Akbani R, Liu Y, Shen H, Robertson AG, Pashtan I, Shen R, et al: Integrated genomic characterization of endometrial carcinoma. Nature 497: 67-73, 2013.

13. Ohga T, Koike K, Ono M, Makino Y, Itagaki Y, Tanimoto M, Kuwano $\mathrm{M}$ and Kohno K: Role of the human Y box-binding protein YB-1 in cellular sensitivity to the DNA-damaging agents cisplatin, mitomycin $\mathrm{C}$ and ultraviolet light. Cancer Res 56: 4224-4228, 1996.

14. Abal M, Llauradó M, Doll A, Monge M, Colas E, González M, Rigau M, Alazzouzi H, Demajo S, Castellví J, et al: Molecular determinants of invasion in endometrial cancer. Clin Transl Oncol 9: 272-277, 2007.

15. Colas E, Perez C, Cabrera S, Pedrola N, Monge M, Castellvi J, Eyzaguirre F, Gregorio J, Ruiz A, Llaurado M, et al: Molecular markers of endometrial carcinoma detected in uterine aspirates. Int J Cancer 129: 2435-2444, 2011

16. Sun P, Xia S, Lal B, Eberhart CG, Quinones-Hinojosa A, Maciaczyk J, Matsui W, Dimeco F, Piccirillo SM, Vescovi AL and Laterra J: DNER, an epigenetically modulated gene, regulates glioblastoma-derived neurosphere cell differentiation and tumor propagation. Stem Cells 27: 1473-1486, 2009.

17. Dewdney SB, Rimel BJ, Thaker PH, Thompson DM Jr, Schmidt A, Huettner P, Mutch DG, Gao F and Goodfellow PJ: Aberrant methylation of the X-linked ribosomal S6 kinase RPS6KA6 (RSK4) in endometrial cancers. Clin Cancer Res 17: 2120-2129, 2011.

18. Bartman AE, Buisine MP, Aubert JP, Niehans GA, Toribara NW, Kim YS, Kelly EJ, Crabtree JE and Ho SB: The MUC6 secretory mucin gene is expressed in a wide variety of epithelial tissues. J Pathol 186: 398-405, 1998.

19. Herbst RS: Review of epidermal growth factor receptor biology. Int J Radiat Oncol Biol Phys 59: 21-26, 2004.

20. Konecny GE, Santos L, Winterhoff B, Hatmal M, Keeney GL, Mariani A, Jones M, Neuper C, Thomas B, Muderspach L and Riehle D: HER2 gene amplification and EGFR expression in a large cohort of surgically staged patients with nonendometrioid (type II) endometrial cancer. Br J Cancer 100: 89-95, 2009. 
21. Ise T, Nagatani G, Imamura T, Kato K, Takano H, Nomoto $M$, Izumi $\mathrm{H}$, Ohmori $\mathrm{H}$, Okamoto $\mathrm{T}$, Ohga $\mathrm{T}$, et al: Transcription factor Y-box binding protein 1 binds preferentially to cisplatin-modified DNA and interacts with proliferating cell nuclear antigen. Cancer Res 59: 342-346, 1999.

22. Albitar L, Pickett G, Morgan M, Wilken JA, Maihle NJ and Leslie KK: EGFR isoforms and gene regulation in human endometrial cancer cells. Mol Cancer 9: 166, 2010.

23. Chon HS, Marchion DC, Xiong Y, Chen N, Bicaku E, Stickles XB Bou Zgheib N, Judson PL, Hakam A, Gonzalez-Bosquet J, et al: The BCL2 antagonist of cell death pathway influences endometrial cancer cell sensitivity to cisplatin. Gynecol Oncol 124: $119-124,2012$
24. Tashiro H, Blazes MS, Wu R, Cho KR, Bose S, Wang SI, Li J, Parsons R and Ellenson LH: Mutations in PTEN are frequent in endometrial carcinoma but rare in other common gynecological malignancies. Cancer Res 57: 3935-3940, 1997. 